\title{
Evolución del impacto de la cardiopatía isquémica en la reincorporación laboral de trabajadores españoles
}

\author{
Evolution of Ischemic Cardiopathy Impact on Spanish Workers on their Return to \\ Work
}

\author{
Ángel García García', Charo Ramos Ramos', Beatriz Toledo Hervás', Víctor Inchausti Sánchez', César González \\ Ramirez² Helena Fernández Bardisa², María Teresa González López ${ }^{3}$ y Rafael Ramos Muñoz ${ }^{4}$ \\ 1. Servicio de Cardiología/Rehabilitación Cardiaca Ibermutuamur Madrid. España. \\ 2. Departamento de Contingencia Común Ibermutuamur. Madrid. España. \\ 3. Departamento de Programas Sanitarios, Proyectos y Atención Especializada Contingencia Común Ibermutuamur. \\ Madrid. España. \\ 4. Dirección Médica de Contingencia Común Ibermutuamur. Madrid. España
}

Recibido: 15-04-15

Aceptado: 08-09-15

\section{Correspondencia}

Ángel García García

Servicio de Cardiología/Rehabilitación Cardiaca Ibermułuamur Madrid. España.

Correo electrónico: AngelGarcia@ibermutuamur.es

Resumen

Introducción: La cardiopatía isquémica es una de las principales causas de morbimortalidad en los países desarrollados dificultando la vuelta al trabajo de estos pacientes. Pretendemos estudiar la evolución de la incapacidad Temporal y Permanente en trabajadores españoles que causan baja por cardiopatía isquémica, así como analizar las variables que influyen en la reincorporación laboral.

Material y métodos: Estudio observacional retrospectivo de cohortes con pacientes menores 65 años que sufrieron un infarto agudo de miocardio (IAM) entre Enero de 2000 y mayo de 2011. Se recogieron datos clínicos, demográficos, socioeconómicos y laborales para analizar su relación con la reincorporación laboral.

Resultados: Se seleccionaron un total de 5067 pacientes, el 94\% varones, y con una edad media de 54,3 años. Un total de 3.679 pacientes $(72,6 \%)$ se reincorporaron a su actividad laboral tras el infarto, mientras que 1261 (24,9\%) obtuvieron la incapacidad laboral permanente. La mayores tasas de incapacidad laboral se observan en los pacientes > 50 años, en los que han sufrido un mayor daño cardiaco (FEVI < 50\%), en aquellos con un mayor número de arterias coronarias obstruidas y los que fueron revascularizados mediante cirugía. En cuanto a las variables socioeconómicas se reincorporan menos los trabajadores con salarios más bajos, los trabajadores por cuenta ajena y los que realizan trabajos en los que predomina la carga física y manual frente a las profesiones en que predomina el trabajo intelectual. No encontramos diferencias con respecto a la tasa de reincorporación entre hombres y mujeres o en función del diagnostico que motivó la baja (síndrome coronario agudo $v s$. angina).

Observamos que la tasa de reincorporación laboral se ha incrementado de forma progresiva en la última década. (Desde tasas de reincorporación del 60\% en el 2000-2002 a tasas superiores al 80\% a partir de 2010) Este incremento se observa en todos los sectores ocupacionales como en todos los tramos etarios en los que se distribuye la muestra

Conclusiones: En nuestro estudio se observa un aumento progresivo en los últimos años en el porcentaje de pacientes que se reincorporan al trabajo. Las variables que mejor predicen la reincorporación 
laboral tras sufrir una baja por cardiopatía isquémica son viables clínicas como la extensión del daño miocárdico (FEVI $<50 \% / F E V I ~>50 \%)$ y el procedimiento de revascularización empleado (percutáneo/cirugía) así como las variables socioeconómicas referentes al tipo de trabajo (autónomo/cuenta ajena), ocupación profesional (carga física/carga intelectual) y la edad del trabajador

Med Segur Trab (Internet) 2015; 61 (240) 325-341

Palabras clave: Infarto de miocardio. Angina de esfuerzo Reincorporación laboral. Incapacidad laboral temporal, Cardiopatía isquémica.

Abstract

Background: Ischemic cardiopathy is one of the main causes of morbidity and mortality in developed countries. It affects the patients quality of life and interferes with their return to work, thus generating a significant economic cost. The aim of our study is to assess labor conditions of patients who suffered an acute myocardial infarction (AMI), highlighting the factors that could have an impact on their return-to-work time.

Materials and metbods: Observational retrospective study with patients under 65 years old who suffered an AMI between January 2000 and May 2011. Data concerning clinical, social, demographic and labor circumstances was collected to better understand its relation with the return to work.

Results: A total of 5.067 patients with Coronary Heart Disease (hereinafter referred to as CHD) were selected. $94 \%$ of the samples were men, under 54.3 years old. 3679 out of 5067 patients (72. 6\%) returned to work after a heart infarct whereas 1261 (24.9\%) did not return, obtaining the permanent incapacity to work. Among the clinical and patient factors, the main determinants were age (mostly <50 years), severity of AMI, poor left ventricular function ( $\mathrm{EF}>50 \% / \mathrm{EF}>50 \%$ ) and revascularization procedures (percutaneous coronary interventions -PCI- and coronary artery bypass graft surgery, CABG). Regarding the economic and jobrelated factors three variables were found to be the strongest predictors of returning to work: job demands (physically demanding jobs vs. intellectually demanding jobs), incomes and job conditions (self-employment vs. employees). There were no significant differences on the returning-to-work rate between men or women or between sick leave due to an acute coronary syndrome or due to an angina.

We observed that the return-to-work activity increased gradually from 2000-2011 (from rates of $60 \%$ in 2000-2002 to over $80 \%$ in 2010). This increase is present in all occupational activities as well as in all age ranges.

Conclusion: In our study we observed a gradual increase of patients returning to work in recent years. The variables used to better predict the return-to-work time in a period of sick leave after a CHD episode are categorized as follows: clinic viable like the extent of myocardial damage (LVEF $<50 \% / \mathrm{LVEF}>50 \%$ ) and the revascularization procedures (Percutaneous coronary intervention (PCI) or coronary artery bypass graft surgery, $C A B G$ ), socioeconomic variables related to the kind of job (self-employment vs. employees) and lastly labor conditions like job demands (physical-demanding jobs vs. intellectual-demanding jobs) or age.

Med Segur Trab (Internet) 2015; 61 (240) 325-341

Key words: Myocardial infarction. Myocardial angina, Return to work. Coronary heart disease. 


\section{INTRODUCCIÓN}

La cardiopatía isquémica es una de las patologías más prevalentes en pacientes en edad laboral en los países desarrollados y es la causa más importante de mortalidad cardiovascular $\left(37,28 \%\right.$ en varones y $23,79 \%$ en mujeres, INE 2007) ${ }^{1}$. Los cambios que la enfermedad coronaria determina en la calidad de vida de los enfermos tienen gran trascendencia no sólo para el bienestar personal sino también para la economía colectiva, generando un importante gasto económico, social y laboral. La cardiopatía isquémica es una de las causas más importantes de incapacidad laboral permanente (I. P.) en mayores de 40 años y la primera causa en número de años de vida laboral perdidos ajustados por discapacidad (AVAD) ${ }^{2}$. Por estos motivos esta patología es objeto de interés de muchos estudios epidemiológicos Aunque la gravedad del IAM constituye un factor esencial en las posibilidades de reincorporación laboral, en los estudios publicados se constata el peso importante de condicionamientos psicológicos y sociales ${ }^{3,4}$. También influye la situación económica y social de cada país, de forma que en los porcentajes de pacientes, en las mismas condiciones patológicas, que vuelven al trabajo tras un episodio de IAM se observan grandes variaciones de unos países a otros ${ }^{5}$. Pretendemos analizar en el presente trabajo las principales variables demográficas, profesionales, clínicas y terapéuticas que influyen, en nuestro medio, en la reincorporación al trabajo tras sufrir infarto de miocardio. Probablemente el mejor parámetro para valorar la "recuperación" de un paciente que ha sufrido enfermedad coronaria (angina o infarto de miocardio) es el retorno del paciente a su trabajo. El que pueda volver a desarrollar normalmente su actividad laboral es probablemente el mejor indicador de la calidad de las intervenciones terapéuticas recibidas y se los procesos de gestión del tiempo necesitado para su recuperación.

Son muy pocos los estudios publicados en nuestro país sobre la repercusión de la cardiopatía isquémica en la vida laboral, tanto en lo referente a la duración de los procesos de incapacidad temporal (I. T.) como al porcentaje de sujetos que se reincorporan al trabajo tras sufrir un infarto ${ }^{6,7}$. La mayoría de estudios publicados incluyen muestras muy pequeñas y con frecuencia están circunscritos a un ámbito local (provincia, comunidad autónoma), por lo que su potencia estadística es limitada ${ }^{8,9}$. Además, la mayoría de los estudios publicados son de los años noventa y, por lo tanto, no recogen los importantes avances terapéuticos desarrollados en la última década en el tratamiento de la cardiopatía isquémica. Por ello consideramos importante disponer de datos que reflejen la situación del impacto laboral de esta enfermedad en los últimos años.

El conocimiento de los factores que influyen en la reincorporación al trabajo tras un IAM puede ayudar a desarrollar modelos de gestión e intervención más eficientes.

El conocimiento de los factores que influyen en la reincorporación al trabajo tras un IAM puede ayudarnos a mejorar los modelos de gestión e intervención más eficientes que eviten prolongar innecesariamente situaciones provisionales como la I. T y contribuyan a reducir las tasas la $\mathrm{I}$. $\mathrm{P}$.

\section{OBJETIVOS}

1. Analizar el impacto que la cardiopatía isquémica supone desde el pdv socioeconómico y laboral: Incapacidad laboral transitoria (I. T.) y permanente (I. P.) asociada a esta enfermedad.

2. Estudiar la variables clínicas, terapéuticas, sociolaborales y demográficas que tienen relación con la duración de la I. T. y la I. P.

3. Comprobar si los avances en los procedimientos terapéuticos desarrollados en la última década en el tratamiento de la cardiopatía isquémica (revascularización percutánea, tratamiento farmacológico, rehabilitación cardiaca) han contribuido de forma significativa a reducir la incapacidad laboral y acortar la duración de la Incapacidad Temporal. 


\section{MATERIAL Y MÉTODOS}

\section{Diseño y población de estudio}

Es un estudio descriptivo, de cohortes, retrospectivo, en el que se incluyen todos los sujetos que causan baja laboral por cardiopatía isquémica desde el 1 enero de 2000 hasta el 31 de mayo de 2011. Todos los trabajadores estaban afiliados a la Seguridad Social y trabajaban en empresas que contrataron la gestión de contingencias comunes y profesionales con Ibermutuamur (Mutua de Accidentes de Trabajo y Enfermedades Profesionales de la Seguridad Social registrada en España con el número n. ${ }^{\circ}$ 274). Se incluyen tanto trabajadores del Régimen General de la Seguridad Social (RGSS) como del Régimen Especial de Autónomos.

Los datos se han obtenido del programa e-sanit@rio+, que contiene toda la información digitalizada relativa a la historia clínica los trabajadores con procesos en baja de Ibermutuamur.

El diagnóstico médico se analizó según el código diagnóstico de la versión 9 de la Clasificación Internacional de Enfermedades y se incluyeron los códigos 410, 411, 412 y 413 de la CIE-9. Se seleccionaron todos los procesos de baja controlada por incapacidad laboral de contingencias comunes y contingencias profesionales en todo el territorio nacional durante los años 2000 al 2011 correspondientes a dichos códigos de la CIE-9. De esta primera selección se eliminaron los registros de pago delegado de duración $<15$ días, ya que es a partir del decimosexto día cuando la Mutua asume el control y seguimiento del proceso. El seguimiento y control de los procesos de incapacidad se realiza por médicos, especialistas en Medicina Familiar y Comunitaria. Desde el 1 enero de 2000 al 31 de mayo de 2011 un total de 7150 pacientes causaron baja laboral por cardiopatía isquémica. Las historias clínicas correspondientes a estos 6819 pacientes fueron posteriormente revisadas una por una por personal sanitario autorizado para acceder al historial de los pacientes con el fin de depurar aquellos casos cuyo diagnostico inicial no fuera confirmado por el diagnostico del informe hospitalario del alta. Tras este cribaje se excluyeron 1.043 pacientes a los que no se les pudo realizar el seguimiento completo por falta de datos (pacientes "sin datos durante el seguimiento") Posteriormente fueron excluidos 265 en los que la coronariografía mostró arterias coronarias sin lesiones ("coronarias sin lesiones", el diagnóstico correspondía a "angina vasoespástica”, puentes intramiocárdicos, miocardiopatía hipertrófica, etc.) 183 casos fueron excluidos por "otros diagnósticos”, 282 casos por duración de la baja $<15$ días. Se excluyeron también a 331 cuyo proceso fue considerado como recaída (pacientes con recaída) por ser un factor que puede distorsionar los resultados y su interpretación. Se excluyeron también a todos aquellos paciente que tenían concedida una incapacidad laboral previa al infarto y/o eran beneficiarios de algún tipo de pensión Finalmente fueron seleccionados 5067 pacientes.

Las altas médicas se confirmaron con el parte de alta extendido por el médico de atención primaria correspondiente.

Los casos de Incapacidad Permanente se confirmaron todos ellos con los expedientes de resolución de Incapacidad Permanente calificados por el Equipo de Valoración de Incapacidades del INSS, por lo tanto son todos ellos casos confirmados.

\section{Variables de interés}

Se analizaron las siguientes variables: reincorporación al trabajo tras la enfermedad (RL), incapacidad permanente (IP), duración media del período de incapacidad temporal (IT).

Las variables independientes se relacionan con variables dependientes clínicas como el diagnostico médico por el que causa baja (angina vs. infarto), procedimiento de revascularizacion coronaria (Cirugía vs ACTP), función sistólica del ventrículo izquierdo 
tras la revascularizacion (FEVI $<50 \%$ vs. FEVI $>50 \%$ ) y con variables sociales como la edad, sexo, el régimen de la seguridad social, profesión-ocupación laboral, retribución económica, comunidad autónoma de residencia de trabajador y el año en el que causó la baja.

Para la profesión se distribuyó a los pacientes en 10 categorías profesionales conforme establece La clasificación nacional de ocupaciones 2011 (CNO-11 aprobada en el Real Decreto 1591/2010, de 26 de diciembre). Con los conductores del grupo II se estable una categoría aparte por las restricciones específicas que la legislación impone a este colectivo. En base a las demandas físicas de la actividad laboral se establecen dos categorías (Trabajo manual, en el que se incluyen ocupaciones en las que predomina la realización de tareas monótonas y repetitivas que requieren fundamentalmente esfuerzo físico y Trabajo intelectual en el que se incluyen los trabajadores que realizan tareas intelectuales).

Para la variable edad se establecen tramos etarios de acuerdo con el siguiente criterio: <30 años; 30-40 años; de 40-50 años, de 50-60 años, 60-65 años y>65 años.

Para su análisis de la retribución económica de los trabajadores se han agrupado en 5 tramos en función de la base reguladora media (50€): hasta $30 €$, de 31 a $50 €$, de 51 a $70 €$, de 71 a $90 €$, y mayor $90 €$.

Para el cálculo de los días transcurridos desde la baja médica de los procesos hasta la solicitud de la propuesta de alta se excluyen los procesos con recaídas para evitar errores en el cálculo.

\section{Análisis estadístico}

En cuanto el análisis de los datos se utilizó el paquete estadístico SPSS (Statistical Package for Social Sciences) versión 15.0 para Windows. Se consideraron diferencias significativas aquellas con un valor de significación inferior a 0,05 ( $\mathrm{p}<0,05)$. Las variables continuas se expresan como media \pm desviación estándar y las variables categóricas como números y porcentajes. Se utilizó el análisis de varianza para las variables continuas y el test de X2 o test exacto de Fisher para las variables categóricas. Para identificar las variables independientes asociadas con la reincorporación laboral se utilizó análisis multivariante que se realizó por medio de la regresión logística. Para el análisis de regresión logística se incluyeron todas las variables significativas, con $\mathrm{p}<0,05$, Para la valorar la magnitud de la asociación se utilizó el cálculo de la odds ratio (OR) y la precisión de la estimación del efecto se midió a través del intervalo de confianza (IC) del 95\% para cada variable.

En todos los análisis, la edad y la base reguladora se incluyeron tanto como variables continuas como por los tramos anteriormente especificados. Para el análisis laboral se distribuyeron a los trabajadores en 11 categoría laborales conforme el grado de demandas de carga física/intelectual de cada ocupación.

Se realizó un estudio descriptivo.

\section{RESULTADOS}

Las características de la población de estudio se muestran en la tabla $n .{ }^{\circ} 1$. La edad media de los sujetos estudiados fue de 54,3 años (DS 8), con un predomino de varones $(94,1 \%)$, como es habitual en esta patología. Con respecto al diagnóstico, la mayoría de las bajas se produjeron por Síndrome Coronario Agudo (SCA) (73,7\%) frente a angina de esfuerzo (26,3\%). En la mayoría de los pacientes había una sola arteria coronaria obstruida $(57,3 \%)$ el 26,3 tenía dos arterias coronarias con lesiones obstructivas y un 15,6\% tenían 3 o más coronarias con obstrucciones significativas. El procedimiento de revascularización más utilizado fue el percutáneo $(82,2 \%)$ frente a la revascularización quirúrgica $(11,9 \%)$. Con respecto al daño miocárdico causado, la mayoría de los pacientes tenía, después del infarto, una función sistólica conservada $(85,2 \%$ tenían FEVI $>50 \%$ frente a un 14,8\% con FEVI $<50 \%$ ). 
Tabla n. ${ }^{\circ}$ 1. Características de la muestra (1)

\begin{tabular}{|c|c|c|c|c|c|}
\hline \multicolumn{6}{|c|}{ CARACTERÍSTICAS DE LA MUESTRA (1) } \\
\hline & $\mathbf{N}$ & $\%$ & & $\mathbf{N}$ & $\%$ \\
\hline \multirow[t]{2}{*}{ N Total } & 5.067 & & Diagnóstico & & \\
\hline & & & Angina & 1.335 & $26,3 \%$ \\
\hline Sexo & & & Infarto & 3.732 & $73,7 \%$ \\
\hline Hombres & 4.778 & $94,2 \%$ & Revascularización & 151 & $3,2 \%$ \\
\hline \multirow[t]{4}{*}{ Mujeres } & 292 & $5,8 \%$ & ACTP & 3.501 & $82,2 \%$ \\
\hline & & & Cirugía & 506 & $11,9 \%$ \\
\hline & & & Fibrinolisis & 50 & $1,2 \%$ \\
\hline & & & No revascularizable & 194 & $4,6 \%$ \\
\hline \multirow[t]{2}{*}{ Edad (X) } & $54,3( \pm 7,6)$ & & FEVI & & \\
\hline & & & $>50 \%$ & 3.366 & $85,2 \%$ \\
\hline FRCV & & & $<50 \%$ & 585 & $14,8 \%$ \\
\hline Tabaco & 3.436 & $67,8 \%$ & N. ${ }^{\circ}$ vasos obstruidos & 49 & $1 \%$ \\
\hline Hipertensión & 2.490 & $49,1 \%$ & 1 vaso & 2.327 & $57,3 \%$ \\
\hline Hipercolesterol & 2.773 & $54,7 \%$ & 2 vasos & 1.068 & $26,3 \%$ \\
\hline Diabetes & 846 & $16,7 \%$ & 3 o $>3$ vasos & 634 & $15,6 \%$ \\
\hline
\end{tabular}

Las características socioeconómicas de la muestra se detallan en la tabla 2. En cuanto al régimen laboral hay un predominio de trabajadores por cuenta ajena $(70,4 \%)$ frente a autónomos (29,6\%), que en líneas generales refleja la distribución de la población laboral en España. Con respecto al tipo de contingencia la mayor parte de las bajas fueron por enfermedad común (CC 89,4\%) frente a un 10,6\% por accidente laboral (CP). Al estudiar las características socioeconómicas, encontramos que la retribución salarial media diaria (base reguladora) fue de 50,15 euros/día (DS 23). Excepto la actividad agraria $(0,9 \%$ de los sujetos) las restantes ocupaciones profesionales descritas en la CNO-11 están suficientemente representadas. Las ocupaciones más representadas son los sectores de industria $(31,3 \%)$, construcción $(11,3 \%)$ y servicios $(14,5 \%)$. Destaca en la muestra el elevado número de conductores (8,9\%), por lo que atendiendo a las especiales características de este colectivo, por las restricciones legislativas con respecto a sus procesos de I. T. e IP consideramos que merece un análisis complementario. Hay un predominio de trabajadores cuya actividad laboral demanda fundamentalmente carga física (trabajadores manuales) frente a aquellos en que predomina la actividad intelectual (trabajado intelectual).

Tabla n. ${ }^{\circ}$ 2. Características socioeconómicas de la muestra (2)

\begin{tabular}{|c|c|c|c|c|c|}
\hline \multicolumn{6}{|c|}{ CARACTERÍSTICAS DE LA MUESTRA (2) } \\
\hline & $\mathbf{N}$ & $\%$ & & $\mathbf{N}$ & $\%$ \\
\hline Régimen Laboral & & & Profesión & & \\
\hline Autónomos & 1.466 & $29,6 \%$ & Directivos & 165 & $3,4 \%$ \\
\hline \multirow[t]{2}{*}{ Cuenta ajena } & 3.481 & $70,4 \%$ & Profesionales & 214 & $4,4 \%$ \\
\hline & & & Intermedios & 463 & $9,5 \%$ \\
\hline Contingencia & & & Administrativos & 492 & $10,1 \%$ \\
\hline C. Común & 4.299 & $89,4 \%$ & Comercio/servicio & 785 & $16,1 \%$ \\
\hline C. Profesional & 509 & $10,6 \%$ & Industria & 1.649 & $33,8 \%$ \\
\hline
\end{tabular}




\begin{tabular}{|c|c|c|c|c|c|}
\hline \multicolumn{6}{|c|}{ CARACTERÍSTICAS DE LA MUESTRA (2) } \\
\hline & $\mathbf{N}$ & $\%$ & & $\mathbf{N}$ & $\%$ \\
\hline Retribución media & $50,15( \pm 23)$ & & Profesión & & \\
\hline$<30 €$ & 335 & $6,7 \%$ & Agricultura & 45 & $0,9 \%$ \\
\hline $30-50 €$ & 2.823 & $56,2 \%$ & Construcción & 585 & $12 \%$ \\
\hline $50-70 €$ & 913 & $18,2 \%$ & Conductores & 476 & $9,7 \%$ \\
\hline $70-90 €$ & 497 & $9,9 \%$ & T. Manual & 3.517 & $72,6 \%$ \\
\hline$>90 €$ & 452 & $9 \%$ & T. Intelectual & 1.330 & $27,4 \%$ \\
\hline$>50 €$ & 1.862 & $37,1 \%$ & & & \\
\hline$<50 €$ & 3.160 & $62,9 \%$ & & & \\
\hline
\end{tabular}

Con respecto a la distribución geográfica (tabla 7) se observa que no es uniforme en todas las comunidades y traduce en primer lugar el grado de implantación de la mutua en las distintas comunidades. Esta circunstancia condiciona la interpretación de los resultados a la hora de comparar unas comunidades con otras. Las comunidades más representadas son Madrid (25,5\%), Andalucía (15,6\%) Murcia (15,8\%) Castilla-León (9,8\%), C. Valenciana (4,7\%), Asturias y Castilla-La Mancha (4,7\%). Con muy poca representación están las comunidades autónomas de País Vasco $(0,4 \%)$ Navarra $(0,2 \%)$, La Rioja $(0,3 \%)$, Aragón $(0,2 \%)$ y Cataluña $(1,3 \%)$.

\section{Análisis de la Reincorporación Laboral}

Tras analizar los datos observamos que $3679(72,6 \%)$ trabajadores se reincorporación al trabajo, mientras que $1261(24,9 \%)$ obtuvieron la incapacidad para su actividad laboral Un total de $59(1,2 \%)$ pacientes fallecieron durante el proceso de baja y $39(0,8 \%)$ pacientes pasaron a situación de jubilación.

Tabla 3. Resultados Globales

\begin{tabular}{lcc}
\hline & RESULTADOS (I) & \\
\hline $\mathrm{N}(\%)$ & 5.079 & $100 \%$ \\
Reincorporación laboral & 3.679 & $72,6 \%$ \\
Incapacidad Permanente & 1.261 & $24,9 \%$ \\
Jubilación & 39 & $0,8 \%$ \\
Fallecimiento & 59 & $1,2 \%$ \\
Duración IT (X, días) & $202( \pm 171)$ & \\
Base reguladora (X) & $49( \pm 23)$ & \\
\hline
\end{tabular}

En el análisis estadístico (tabla 4) encontramos que la tasa de reincorporación laboral se asocia de forma estadísticamente significativa con la edad (cuanto mayor es la edad del trabajador menor es la probabilidad de reincorporación) y con las siguientes variables clínicas: la extensión del daño miocárdico (FEVI $>50 \% /<50 \%$ ) a mayor daño menor probabilidad de reincorporación $(78,7 \%$ vs $54,2 \%$ p $<0,000)$, con el procedimiento de revascularización: se reincorporan más los pacientes sometidos a revascularización percutánea $(76,1 \%$ para ICP vs. $64,1 \%$ para $\mathrm{Cx}$ p <0,000), con el número de arterias coronarias obstruidas: se reincorpora el $76,9 \%$ de los pacientes con $<3$ coronarias obstruidas frente al $60,3 \%$ de pacientes con $>3$ arterias afectas ( $<<0,000)$, Sin embargo no hemos encontrado diferencias significativas con respecto al diagnóstico: se reincorpora el $74,7 \%$ de los pacientes que causan baja por infarto de miocardio y el $73,5 \%$ de los que causan baja por angina de esfuerzo $(p<0,209)$ Tampoco hemos encontrado diferencias 
estadísticamente significativas en cuanto al sexo (se reincorporan el 74,4\% de los hombres y el $78,2 \%$ de las mujeres $\mathrm{p}=0,337)$.

Tabla 4. Resultados: variables clínicas

\begin{tabular}{|c|c|c|c|c|}
\hline \multicolumn{5}{|c|}{ RESULTADOS (II) } \\
\hline & Reincorporación & Incapacidad & $\mathbf{P}$ & Total \\
\hline \multicolumn{5}{|l|}{ Sexo } \\
\hline Hombres & $3.434(74,4 \%)$ & $1.180(25,6 \%)$ & 0,337 & $4.618(94,4 \%)$ \\
\hline Mujeres & $215(78,2 \%)$ & $60(21,6 \%)$ & & $275(5,6 \%)$ \\
\hline Edad (X) & & & 0,000 & $54,32( \pm 7,6)$ \\
\hline$<30 \mathrm{a}$ & $2(100 \%)$ & & & \\
\hline $30-40 a$ & $146(88 \%)$ & $20(12 \%)$ & & $166(3,4 \%)$ \\
\hline $40-50 a$ & $949(83,2 \%)$ & $191(16,8 \%)$ & & $1.140(23,3 \%)$ \\
\hline $50-60 a$ & $1.690(73,7 \%)$ & $604(36,3 \%)$ & & $2.294(46,6 \%)$ \\
\hline$>60 \mathrm{a}$ & $878(66,5 \%)$ & $443(33,5 \%)$ & & $1.321(26,8 \%)$ \\
\hline$>50 \mathrm{a}$ & $2.419(70,5 \%)$ & $1.010(29,5 \%)$ & 0,000 & $3.429(69,7 \%)$ \\
\hline$<50 a$ & $1.240(83,3 \%)$ & $248(16,7 \%)$ & & $1.488(30,3 \%)$ \\
\hline \multicolumn{5}{|l|}{ Diagnóstico } \\
\hline IAM & $2.717(74,7 \%)$ & $818(25,3 \%)$ & 0,209 & $3.635(73,8 \%)$ \\
\hline Angina & $951(73,5 \%)$ & $342(26,5 \%)$ & & $1.293(26,2 \%)$ \\
\hline \multicolumn{5}{|c|}{ N. ${ }^{o}$ vasos con lesiones } \\
\hline 1 & $1.841(80,6 \%)$ & $444(19,4 \%)$ & 0,000 & $2.285(57,5 \%)$ \\
\hline 2 & $728(68,7 \%)$ & $331(31,3 \%)$ & & $1.059(26,4 \%)$ \\
\hline $30>3$ & $384(60,1 \%)$ & $251(39,9 \%)$ & & $635(15,8 \%)$ \\
\hline$<3$ & $2.569(76,9 \%)$ & $775(23,1 \%)$ & 0,000 & $3.344(84,1 \%)$ \\
\hline$>3$ & $384(60,3 \%)$ & $251(39,1 \%)$ & & $635(15,9 \%)$ \\
\hline \multicolumn{5}{|c|}{ Revascularización } \\
\hline ACTP & $2.622(76,1 \%)$ & $708(21,3 \%)$ & 0,000 & $3.445(87,7 \%)$ \\
\hline Cirugía & $311(64,1 \%)$ & $174(35,9 \%)$ & & $485(12,3 \%)$ \\
\hline \multicolumn{5}{|l|}{ FEVI } \\
\hline$>50 \%$ & $2.613(78,7 \%)$ & $565(20,1 \%)$ & 0,000 & $3.321(85,1 \%)$ \\
\hline$<50 \%$ & $306(54,2 \%)$ & $259(45,8 \%)$ & & $565(14,5 \%)$ \\
\hline
\end{tabular}

En la tabla 5 se exponen los resultados referentes a los factores sociolaborales Encontramos diferencias estadísticamente significativas para el tipo de contingencia (se reincorporan más los pacientes cuyo proceso se califica de enfermedad común que como accidente laboral $(73,4 \%$ vs. $64,3 \% \mathrm{p}<0,000))$.

También en lo que se refiere al régimen laboral hay una relación estadísticamente significativa: se reincorporan más los trabajadores autónomos que los trabajadores por cuenta ajena $(83,5 \%$ vs. $69,3 \% \mathrm{p}<0,000)$. 
Tabla 5. Resultados: variables socioeconómicas

\begin{tabular}{|c|c|c|c|c|}
\hline \multicolumn{5}{|c|}{ RESULTADOS (II) } \\
\hline & Reincorporación & Incapacidad & $\mathbf{P}$ & Total (\%) \\
\hline \multicolumn{5}{|l|}{ Régimen Laboral } \\
\hline Autónomos & $1.214(84,7 \%)$ & $220(15,3 \%)$ & 0,000 & $1.434(29,8 \%)$ \\
\hline C. ajena & $2.384(70,4 \%)$ & $1.000(29,6 \%)$ & & $3.384(70,2 \%)$ \\
\hline \multicolumn{5}{|l|}{ Contingencia } \\
\hline C. Común & $3.134(75 \%)$ & $1.044(25 \%)$ & 0,000 & $4.178(89,3 \%)$ \\
\hline C. Profesional & $327(65,5 \%)$ & $172(34,5 \%)$ & & $499(10,7 \%)$ \\
\hline \multicolumn{5}{|l|}{ Base Reguladora } \\
\hline$>50 €$ & $1.450(79,9 \%)$ & $365(20,1 \%)$ & 0.000 & $1.815(37,1 \%)$ \\
\hline$<50 €$ & $2.184(71,1 \%)$ & $889(28,9 \%)$ & & $3.073(62,9 \%)$ \\
\hline$<30 €$ & $247(76,5 \%)$ & $76(23,5 \%)$ & & $323(6,6 \%)$ \\
\hline $30-50 €$ & $1.936(70,4 \%)$ & $813(29,6 \%)$ & & $2.749(56,3 \%)$ \\
\hline $50-70 €$ & $655(75,1 \%)$ & $221(24,9 \%)$ & & $886(18,1 \%)$ \\
\hline $70-90 €$ & $385(79,9 \%)$ & $97(20,1 \%)$ & & $482(9,9 \%)$ \\
\hline$>90 €$ & $400(89,5 \%)$ & $47(10,5 \%)$ & & $447(9,1 \%)$ \\
\hline \multicolumn{5}{|l|}{ Tipo de Trabajo } \\
\hline Manual & $2.390(69,6 \%)$ & $1.045(30,4 \%)$ & 0,000 & $3.435(72,6 \%)$ \\
\hline Intelectual & $1.121(86,3 \%)$ & $178(13,7 \%)$ & & $1.299(27,4 \%)$ \\
\hline \multicolumn{5}{|l|}{ Ocupación Laboral } \\
\hline Directivos & $141(87,6 \%)$ & $20(13,4 \%)$ & 0,000 & $161(3,4 \%)$ \\
\hline Profesionales & $193(92,3 \%)$ & $16(7,7 \%)$ & & $209(4,4 \%)$ \\
\hline Intermedios & $372(82,9 \%)$ & $77(17,1 \%)$ & & $449(9,4 \%)$ \\
\hline Administrativos & $418(86,4 \%)$ & $66(13,6 \%)$ & & $484(10,1 \%)$ \\
\hline Comercio/servicios & $581(75,8 \%)$ & $185(24,2 \%)$ & & $766(16,1 \%)$ \\
\hline Industria & $1.109(68,5 \%)$ & $511(31,5 \%)$ & & $1.620(34 \%)$ \\
\hline Conductores & $327(70,8 \%)$ & $135(29,2 \%)$ & & $462(9,7 \%)$ \\
\hline Construcción & $367(64,7 \%)$ & $200(35,5 \%)$ & & $567(11,9 \%)$ \\
\hline Agrario & $24(55,8 \%)$ & $19(44,2 \%)$ & & $43(0,9 \%)$ \\
\hline
\end{tabular}

Con respecto a las variables socioeconómicas, tanto la retribución económica como la ocupación laboral se relacionan también de forma estadísticamente significativa con la vuelta al trabajo. Los trabajadores con mayores ingresos se incorporan más $(79,9 \%$ de los que ganan $>50$ euros/día frente a un $71,1 \%$ de los que ganan $<50$ euros/día p $<0,000$ ) Observamos también cómo la tasa de reincorporación aumenta a medida que se incrementa la renta salarial (desde el 70,4\% en los que perciben salarios entre 30-50 euros al $89,5 \%$ de los mejor remunerados). Los pacientes cuya actividad laboral requiere una mayor carga física y/o menor carga intelectual (trabajo manual) se reincorporan en menor proporción que los trabajadores con cargos directivos o actividad profesional (trabajo intelectual) $(69,6 \%$ vs. $83,6 \% \mathrm{p}<0,000)$. En cuanto a la ocupación profesional se reincorpora el $64,7 \%$ de trabajadores de la construcción, el 55,8\% de agricultores, un $68,5 \%$ de industria, el 75,8\% de servicios, el $86,4 \%$ de administrativos y tasas del $92,3 \%$ y $86,7 \%$ para profesionales y cargos directivos respectivamente.

Respecto a la edad (tabla 6) se observa una clara tendencia decreciente con un descenso progresivo del porcentaje de reincorporación a medida que aumenta la edad del trabajador. Esta tendencia se mantiene para todas las variables tanto clínicas como sociolaborales. 
Tabla 6. Resultados por categorías etarias

\begin{tabular}{lccccc}
\hline \multicolumn{5}{c}{ REINCORPORACIÓN LABORAL POR EDADES } \\
\hline Reincorporación & $\mathbf{1 4 6}(86,9 \%)$ & $946(81,8 \%)$ & $1.693(72,4 \%)$ & $878(63,4 \%)$ & 0,000 \\
Incapacidad & $20(11,9 \%)$ & $191(16,5 \%)$ & $604(25,8 \%)$ & $443(32 \%)$ & \\
Jubilación & 0 & $1(0,1 \%)$ & 0 & $38(2,7 \%)$ & \\
Fallecimiento & $2(1,2 \%)$ & $11(1 \%)$ & $23(1 \%)$ & $20(1,4 \%)$ & \\
\hline Total & $168(3,3 \%)$ & $1.156(22,9 \%)$ & $2.337(46,3 \%)$ & $1.385(27,4 \%)$ & \\
\hline Duración IT & $193( \pm 119)$ & $187( \pm 130)$ & $201( \pm 133)$ & $224( \pm 143)$ & 0,000 \\
Base Reguladora & $45,6( \pm 22)$ & $46,8( \pm 22)$ & $50,6( \pm 24)$ & $49( \pm 23)$ & 0.000 \\
\hline
\end{tabular}

Con respecto a la distribución geográfica (tabla 7) nos encontramos con una población proporcional a la implantación de Ibermutuamur en dicha comunidad. Por esta razón en algunas autonomías los datos se deben valorar con precaución dada la escasa representatividad de la muestra. No obstante y considerando aquellas autonomías con una representación suficiente, observamos diferencias importantes en lo referente a la incorporación laboral entre unas comunidades y otras: en este sentido la comunidades de Madrid, Castilla-León, Canarias y Alicante son las que tienen un mayor porcentaje de reincorporación laboral $(>80 \%)$. En cambio Andalucía y Asturias destacarían por su baja tasa de incorporación laboral $(<70 \%)$.

Tabla 7. Resultados por Comunidades Autónomas

\begin{tabular}{|c|c|c|c|c|c|}
\hline $\begin{array}{l}\text { COMUNIDAD } \\
\text { AUTÓNOMA }\end{array}$ & REINCORPORACIÓN & INCAPACIDAD & JUBILACIÓN & FALLECIMIENTO & TOTAL \\
\hline Andalucía & $448(57,2 \%)$ & $315(40,2 \%)$ & $2(0,3 \%)$ & $10(1,5 \%)$ & $783(15,5 \%)$ \\
\hline Aragón & $8(80 \%)$ & $2(20 \%)$ & $0(0,0)$ & $0(0,0 \%)$ & $10(0,2 \%)$ \\
\hline Asturias & $159(67,4 \%)$ & $72(30,5 \%)$ & $2(1,1 \%)$ & $1(0,5 \%)$ & $236(4,7 \%)$ \\
\hline Baleares & $20(69 \%)$ & $7(24,1 \%)$ & $0(0.0 \%)$ & $1(4,5 \%)$ & $29(0,6 \%)$ \\
\hline Cantabria & $14(60,9 \%)$ & $9(39,1 \%)$ & $0(0.0 \%)$ & $0(0,0 \%)$ & $23(0.5 \%)$ \\
\hline Canarias & $66(80,5 \%)$ & $12(14,6 \%)$ & $0(0.0 \%)$ & $0(0,0 \%)$ & $82(1,6 \%)$ \\
\hline Castilla-León & $402(80,4 \%)$ & $86(17,2 \%)$ & $5(1,2 \%)$ & $4(0,9 \%)$ & $500(9,9 \%)$ \\
\hline Castilla-La Mancha & $176(73,9 \%)$ & $57(23,9 \%)$ & $1(0.5 \%)$ & $1(0.5 \%)$ & $238(4,7 \%)$ \\
\hline Valenciana & $546(76,9 \%)$ & $140(19,7 \%)$ & $11(1,5 \%)$ & $9(1,3 \%)$ & $240(4,7 \%)$ \\
\hline Cataluña & $42(64,6 \%)$ & $22(33,8 \%)$ & $0(0.0 \%)$ & $0(0,0 \%)$ & $65(1,3 \%)$ \\
\hline Ceuta & $1(50 \%)$ & $1(50 \%)$ & $0(0.0 \%)$ & $0(0,0 \%)$ & $2(0,0 \%)$ \\
\hline Extremadura & $68(70,1 \%)$ & $25(25,8 \%)$ & $0(0.0 \%)$ & $0(0,0 \%)$ & $97(1,9 \%)$ \\
\hline Galicia & $110(71,9 \%)$ & $40(26,1 \%)$ & 0 & $2(1,3 \%)$ & $153(3 \%)$ \\
\hline Madrid & $1.056(81,9 \%)$ & $201(15,6 \%)$ & $8(0,7 \%)$ & $14(1,3 \%)$ & $1.290(25,5 \%$ \\
\hline Murcia & $526(65,6 \%)$ & $265(33 \%)$ & $5(0,8 \%)$ & $1(0,2 \%)$ & $802(15,8 \%)$ \\
\hline Navarra & $7(87,5 \%)$ & $0(0,0 \%)$ & $0(0,0 \%)$ & $0(0,0 \%)$ & $8(0,2 \%)$ \\
\hline P. Vasco & $16(72,7 \%)$ & $3(17,6 \%)$ & $0(0,0 \%)$ & $2(4,1 \%)$ & $22(0,4 \%)$ \\
\hline Rioja & $12(70,6 \%)$ & $3(18,8 \%)$ & $2(12,5 \%)$ & $1(6,3 \%)$ & $17(0,3 \%)$ \\
\hline TOTAL & $3.186(74,5 \%)$ & $946(22,1 \%)$ & $31(0,7 \%)$ & $49(1,1 \%)$ & 4.277 \\
\hline
\end{tabular}

En la tabla 8 se puede observar la evolución que ha tenido la tasa de reincorporación laboral tras un evento isquémico cardiaco, a lo largo de los últimos 10 años. Es de notar 
el aumento progresivo que se observa en la última década del porcentaje de trabajadores que vuelven trabajar después de sufrir un evento coronario: desde tasas del 60,6\% en el año 2000 hasta tasas $>75 \%$ a partir de 2007 . Este incremento en el retorno al trabajo se acompaña de forma paralela de un descenso también estadísticamente significativo de la media de días de baja por I. T. debidos a esta enfermedad (desde una duración media de 259 días en el año 2000 a duraciones de <200 días a partir de 2007.

Tal como ocurría en el caso de la edad esta tendencia creciente de la tasa de reincorporación se observa para todas las variables estudiadas tanto clínicas como socioeconómicas y para todas las ocupaciones tanto las de alta carga física como para las actividades intelectuales.

Tabla 8. Resultados por año en el que causa la baja

\begin{tabular}{lcccccccccccc}
\hline \multicolumn{10}{c}{ EVOLUCIÓN DE LA REINCORPORACIÓN LABORAL 2000-2011 } \\
\hline & 2000 & 2001 & 2002 & 2003 & 2004 & 2005 & 2006 & 2007 & 2008 & 2009 & 2010 & 2011 \\
\hline Reincorporación & 172 & 227 & 294 & 298 & 356 & 392 & 368 & 221 & 228 & 390 & 468 & 209 \\
& $(60,6 \%)$ & $(60,1 \%)$ & $(71,2 \%)$ & $(68 \%)$ & $(72,2 \%)$ & $(73,7 \%)$ & $(72 \%)$ & $(74,2 \%)$ & $(75,8 \%)$ & $(73,3 \%)$ & $(82,1 \%)$ & $(85,7 \%)$ \\
Incapacidad & 103 & 138 & 106 & 121 & 127 & 127 & 131 & 69 & 84 & 126 & 98 & 31 \\
& $(36,3 \%)$ & $(36,5 \%)$ & $(25,7 \%)$ & $(27,6 \%)$ & $(25,8 \%)$ & $(23,9 \%)$ & $(25,6 \%)$ & $(23,2 \%)$ & $(22,1 \%)$ & $(23,7 \%)$ & $(17,2 \%)$ & $(12,7 \%)$ \\
Jubilación & 30 & 3 & 4 & 6 & 3 & 3 & 1 & 2 & 3 & 7 & 3 & 1 \\
& $(1,1 \%)$ & $(0,8 \%)$ & $(1 \%)$ & $(1,4 \%)$ & $(0,6 \%)$ & $(0,6 \%)$ & $(0,2 \%)$ & $(0,7 \%)$ & $(0,8 \%)$ & $(1,3 \%)$ & $(0,5 \%)$ & $(0,4 \%)$ \\
Fallecimiento & 3 & 5 & 6 & 8 & 4 & 7 & 7 & 5 & 2 & 9 & 1 & 2 \\
& $(1,1 \%)$ & $(1,3 \%)$ & $(1,5 \%)$ & $(1,8 \%)$ & $(0,8 \%)$ & $(1,3 \%)$ & $(1,4 \%)$ & $(1,7 \%)$ & $(0,5 \%)$ & $(1,7 \%)$ & $(0,2 \%)$ & $(0,8 \%)$ \\
\hline Total & 284 & 384 & 413 & 438 & 493 & 525 & 511 & 298 & 380 & 532 & 361 & 244 \\
& $(5,6 \%)$ & $(9 \%)$ & $(8,1 \%)$ & $(8,6 \%)$ & $(9,7 \%)$ & $(12,4 \%)$ & $(10,1 \%)$ & $(5,9 \%)$ & $(7,5 \%)$ & $(10,5 \%)$ & $(8,5 \%)$ & $(4,8 \%)$ \\
\hline Duración IT & 259 & 222 & 208 & 209 & 212 & 213 & 202 & 198 & 183 & 188 & 190 & 167 \\
& $( \pm 151)$ & $( \pm 152)$ & $( \pm 131)$ & $( \pm 141)$ & $( \pm 138)$ & $( \pm 138)$ & $( \pm 131)$ & $( \pm 126)$ & $( \pm 126)$ & $( \pm 131)$ & $( \pm 127)$ & $( \pm 126)$ \\
Base Reguladora & 43,4 & 41,4 & 45 & 45 & 47 & 48 & 48 & 52 & 53 & 54 & 56 & 56 \\
& $( \pm 19)$ & $( \pm 19)$ & $( \pm 20)$ & $( \pm 19)$ & $( \pm 22)$ & $( \pm 22)$ & $( \pm 22)$ & $( \pm 25)$ & $( \pm 25)$ & $( \pm 26)$ & $( \pm 26)$ & $( \pm 26)$ \\
\hline
\end{tabular}

Figura 1. Evolución de la reincorporación laboral en pacientes que causan baja por cardiopatía isquémica desde el año 2000 a 2011

\section{Evolución de la incorporación laboral}

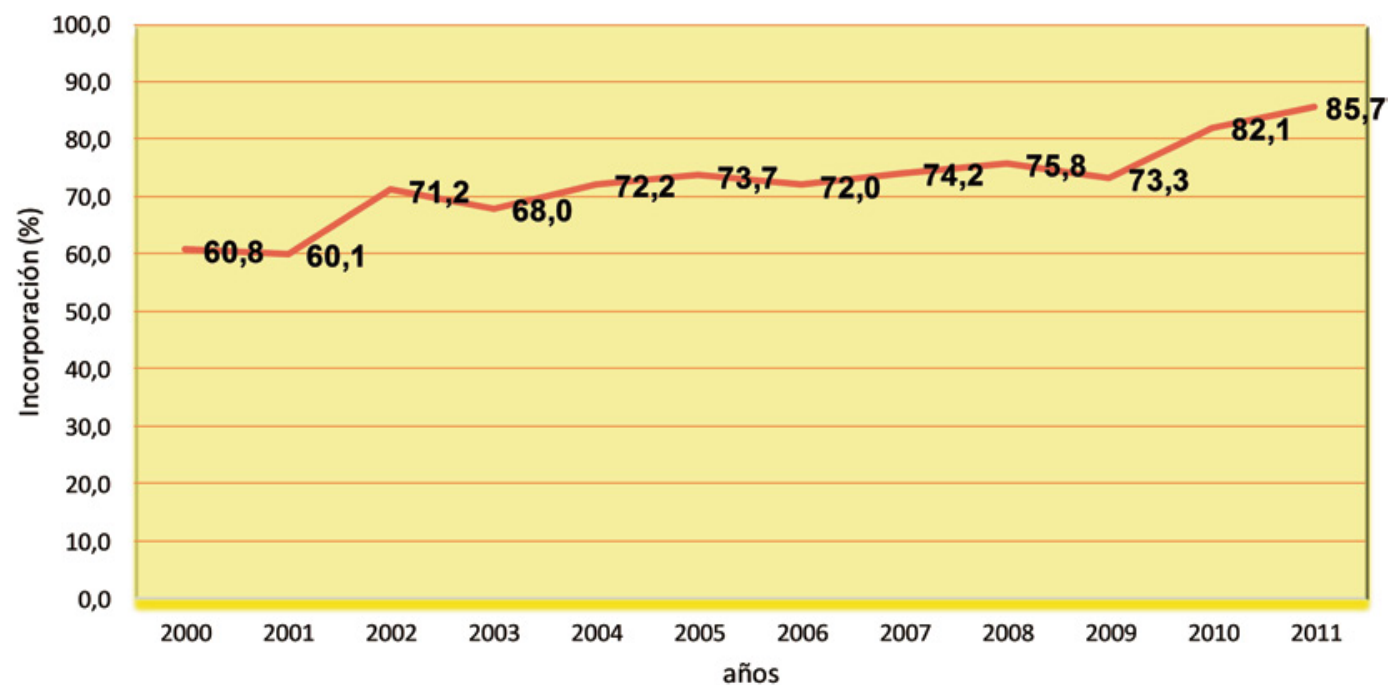

En el análisis de regresión simple se encontraron diferencias estadísticamente significativas con respecto a la reincorporación laboral para las siguientes variables clinicas: FEVI < 50\%/FEVI > 50\% (56,3\% vs. 79,8\% p < 0,000 IC 95\%), para el procedimiento 
de revascularización ICP/cirugía (76,3\% vs. 64,2\% p < 0,000 IC 95\%) y para en número de arterias coronarias obstruidas $(>3 /<3$ arterias obstruidas $77,6 \%$ vs. $60,3 \% \mathrm{p}<0,000$ IC 95\%). No se encontraron diferencias significativas para el diagnóstico (angina vs. infarto) $(74,7 \%$ vs. $73,5 \%$ p $<0,209$ IC $95 \%)$ ni para el sexo $(74,4 \%$ hombres vs. $78,2 \%$ mujeres $\mathrm{p}$ $<0,337$ IC 95\% ) Con respecto a las variables socioeconómicas observamos diferencias significativas para la retribución salarial (base reguladora $>50 /<50$ euros) $(79,9 \%$ vs. $71,2 \%$ p < 0,000 IC 95\%), para el régimen laboral (autónomo vs cuenta ajena: $84,8 \%$ vs. 74,9\% p $<0,000$ IC 95\%) y para el tipo de contingencia (contingencia común vs. contingencia profesional: $75 \%$ vs. $65,2 \%$ p < 0,000 IC 95\%) y para el tipo de ocupación laboral (trabajo manual vs. trabajo intelectual: $69,8 \%$ vs. $86,3 \%$ p $<0,000$ IC 95\%).

Los resultados del análisis de regresión logística se exponen en la tabla 9. En el análisis se encontró que las variables que mejor predicen la reincorporación laboral de los pacientes isquémicos son dos variables clínicas como la extensión del daño miocárdico (FEVI $<50 \% / F E V I>50 \%$ p $<0,000$ IC 95\% 2,521-3,978) y el procedimiento de revascularización (ACTP vs. Cx: p < 0,000 IC 95\% 1,282-2,162); dos variables laborales como el tipo de actividad laboral (trabajo manual vs. trabajo intelectual: ( $p<0,000$ IC 95\% 2,110-3,385) y el régimen laboral (autónomo vs. cuenta ajena: $\mathrm{p}<0,000$ IC 95\% 1,571-2,401) y la edad ( $\mathrm{p}<0,000$ IC 95\% 1,706-2,585). También se encontrón un valor predictivo, pero más débil para variables como retribución económica (base reguladora: p < 0,000 IC 95\% 1,2017-1,800), tipo de contingencia (Común vs. C profesional: $\mathrm{p}<0,000$ IC 95\% 1,1822,038). Sin embargo no se encontró valor predictivo para el sexo: $\mathrm{p}<0,972$ IC 95\% 0,6401,476) ni para el diagnóstico (angina de esfuerzo vs. infarto: $p<0,802$ IC 95\% 0,6500,989 .

Tabla 9. Factores predictores de le reincorporación laboral

\begin{tabular}{lccc}
\hline \multicolumn{4}{c}{ REGRESIÓN LOGÍSTICA } \\
\hline \multicolumn{1}{c}{ Variables } & ODS RATIO & I. C. $\quad(95 \%)$ & p \\
\hline Edad & 2,100 & $1,706-2,585$ & 0,000 \\
Sexo (H/M) & 0,972 & $0,640-1,476$ & 0,893 \\
Diagnóstico (Angina/Infarto) & 0,802 & $0,650-0,989$ & 0,039 \\
Revascularización (ACTP/Cx) & 1,665 & $1,282-2,162$ & 0,000 \\
FEVI (>50\% vs <50\%) & 3,167 & $2,521-3,978$ & 0,000 \\
Contingencia (CC/CP) & 1,552 & $1,182-2,038$ & 0,002 \\
Ocupación (Intelectual/Manual) & 2,672 & $2,110-3,385$ & 0,000 \\
Autónomo/T. cuenta ajena & 1,942 & $1,571-2,401$ & 0,000 \\
Base reguladora & 1,480 & $1,217-1,800$ & 0,000 \\
\hline
\end{tabular}

\section{DISCUSIÓN}

El presente estudio incluye la mayor muestra de pacientes de los publicados hasta ahora en nuestro país, con representación a nivel nacional, incluyendo pacientes de todas las comunidades autónomas. Por el período de inclusión de pacientes (Enero de 2000 a Mayo de 2011) entendemos que la población incluida en el estudio recoge los beneficios aportados por la generalización de los nuevos procedimientos de revascularización coronaria (generalización de la ICP primaria y del uso de Stents farmacoactivos...) y tratamientos farmacológicos (nuevos fármacos antiagregantes, estatinas...) $)^{10,11}$.

La tasa media de reincorporación laboral encontrada en nuestro estudio $(72,6 \%)$ es sensiblemente mayor que la observada en los estudios previos publicados en nuestro país (63.2\% en el estudio de Sobrino et al. ${ }^{12}$, 40\% en el estudio de Expósito Tirado et al. ${ }^{6}$ y $56,6 \%$ en el estudio de Gutiérrez Morlote ${ }^{9}$ ). Pero, sobre todo, destaca la continua y 
progresiva mejora de las tasas de reincorporación laboral observadas a lo largo de los últimos diez años (tabla 8), que nos ha permitido pasar desde porcentajes de reincorporación del 60\% en el año 2000 a porcentajes $>75 \%$ a partir de 2008).

Este incremento progresivo de la tasa de reincorporación laboral se observa en relación a todas las variables estudiadas incluyendo aquellas que tradicionalmente se han asociado a incapacidad laboral, como la edad y el trabajo manual vs. intelectual. Mejora de forma sensible la tasa de incorporación de los sujetos con categoría profesional más baja y ocupación en sectores laborales más duros como construcción, agrícola e industrial. Incluso en los pacientes $>50$ años se observa esta mejoría, manteniendo tasas de reincorporación $>65 \%$. Estos datos nos acercan más a la media de algunos países de nuestro entorno y Estados Unidos (EEUU). En EEUU las tasas de reincorporación laboral generalmente están en torno al 80\% varían entre el 63-94\%, según los estudios publicados ${ }^{5,13,14}$, en Bélgica se han publicado tasas entre el $83 \%-87 \%{ }^{15,16}$, en Francia del $76 \%$ en el estudio de Isaaz ${ }^{17}$ o incluso mejorando las publicadas por algunos países europeos $62,2 \%$ en el estudio de Mitag, en Alemania ${ }^{18}$, 58\% en el estudio de Hämäläinen en Finlandia ${ }^{5}$, y entre 50-60\% en Polonia $^{19}$.

Es importante tener en cuenta al comparar las tasas de reincorporación entre los distintos países que una parte de las diferencias observadas están relacionadas con los diferentes períodos de seguimiento de los estudios, variaciones de edad de los grupos, así como también a los diferentes sistemas de cobertura social de los distintos países. También es importante considerar que muchos de los resultados se basan en datos de grupos de pacientes relativamente pequeños.

En algunos estudios previos publicados se observa que las variables clínicas tienen un menor impacto en la tasa de reincorporación que las variables socioeconómicas y ocupacionales ${ }^{3,16,20-23}$. Sin embargo en nuestro estudio observamos que las variables clínicas cobran una mayor relevancia, y el predictor más potente de la vuelta al trabajo es la extensión del daño miocardico medido por la FEVI. La probabilidad de que un paciente con FEVI $>50 \%$ se reincorpore es tres veces mayor que los sujetos con FEVI $<50 \%$. El procedimiento de revascularización empleado también es un buen predictor. La revascularización quirúrgica conlleva una mayor probabilidad de incapacidad laboral que la revascularización percutánea. El hecho de que no hayamos encontrado diferencias significativas entre los pacientes que causan baja por angina de esfuerzo y los que lo hacen por infarto de miocardio probablemente es debido a que los pacientes con angina tenían un mayor número de vasos obstruidos (el 20,6\% tenían>3 vasos vs 11,9\% en el caso del infarto) y sufrieron en un mayor porcentaje revascularización quirúrgica (19,3\% vs $10 \% \mathrm{p}<0.000)$.

En cuanto a las variables socioeconómicas la edad es un predictor potente y así aparece en la mayoría de los estudios publicados ${ }^{17,24-27}$. El riesgo de tener una incapacidad permanente se incrementa de forma significativa a partir de los 50 años $(80,3 \%$ en $<50$ años y 70,5\% en> 50 años). Observamos como el porcentaje de incapacidad laboral permanente se incrementa de forma gradual y constante con la edad. La incidencia de IP a partir de los 50 años es muy superior a la incidencia en menores de 50 años. No obstante, en nuestro estudio, se observa que ha ido disminuyendo desde el año 2000 al 2011. Este hecho pone de manifiesto cómo en la medida en que la edad aumenta son más frecuentes los problemas de salud de carácter crónico, lo que lógicamente incrementaría el riesgo de una IP.

Los trabajadores autónomos se incorporan antes y en mayor proporción que los asalariados $(84,7 \%$ vs. $70,4 \%)$ probablemente debido a la distinta cobertura económica y social que en nuestro país existe para ambos colectivos. Los trabajadores con mayor retribución económica $(79,9 \%$ vs. $71,1 \%)$ y aquellos que realizan una actividad laboral en la que prima la actividad intelectual se incorporan también en mayor proporción que aquellos cuya actividad laboral es manual (86,3\% vs. 69,6\%). Ambas variables, el trabajo autónomo y la retribución económica, destacan también como predictores de IP en otros procesos diagnósticos diferentes de la cardiopatía isquémica ${ }^{29,30}$. 
En el caso de los conductores, la tasa de reincorporación laboral $(69,2 \%)$ es sensiblemente inferior al sector servicios, con quien comparte el mismo grupo en la CNO11. Esto es debido, como hemos comentado anteriormente a las restricciones que la legislación de tráfico impone para la renovación de la licencia a este colectivo tras sufrir un infarto o implante de dispositivos (DAI, DAI-RCT) ${ }^{28}$.

Destaca el bajo porcentaje de reincorporación observado en el sector agricultura (se reincorpora el 55,8\%) frente a las otras ocupaciones laborales (72\%). En parte puede ser explicado porque esta ocupación implica unas condiciones de trabajo de alto riesgo para la salud y, por otra, por ser poblaciones en proceso de envejecimiento con respecto a otros regímenes, todo lo cual hace esperable una mayor incidencia de IP.

Finalmente, con respecto al análisis por CCAA, las diferencias observadas entre las mismas coinciden también con las observadas para otros procesos diagnósticos ${ }^{29,30}$ y es posible que se deban a la diferente distribución de las actividades económicas (predominio del sector servicios en Madrid frente a sectores agrario en Andalucía y Murcia o minero en la comunidad de Asturias), aunque precisaría de un análisis más profundo. No obstante, un hecho destacable desde el 2000 al 2011 es que se observa una convergencia entre todas las CCAA, lo que podría ser debido a una creciente uniformidad en los procedimientos de gestión y la aplicación de los criterios de la evaluación de la IP por parte de los Equipos de Valoración de Incapacidades de INSS (EVIS).

Con respecto a la reducción progresiva de las tasas de incapacidad observadas, dos son los posibles factores pueden explicarla:

1. Los cambios regulatorios y normativos introducidos en los últimos años en la legislación de la Incapacidad, la ampliación de la colaboración de las mutuas con la Seguridad Social en la gestión de la IT común (1994) y la posibilidad de que los médicos del INSS extiendan altas a efectos económicos (1998) ${ }^{29-31}$. Estas medidas, orientadas a controlar el gasto, entendemos que pueden haber contribuido a una reducción tanto de la tasa de incapacidad como de la duración de los tiempos de baja laboral.

2. En segundo lugar, en las dos últimas décadas hemos asistido en nuestro país a un desarrollo espectacular de los procedimientos de revascularización coronaria y tratamiento farmacológico en el campo de la cardiopatía isquémica Actualmente las tasas de revascularización percutánea inmediata tras el infarto agudo de miocardio (ICP primaria) se han generalizado e incrementado de forma exponencial en casi todas las comunidades de nuestro país, con la consiguiente reducción del daño miocárdico y mejoría pronostica del paciente ${ }^{32}$. La revascularización percutánea (ACTP) ha permitido acortar el ingreso hospitalario a menos de una semana en la mayoría de los casos (en el caso de la angina a 24-48 horas), disminuyendo, además tanto la mortalidad intrahospitalaria y a los 6 meses como las recaídas tempranas ${ }^{32,33}$. La revascularización quirúrgica, más incapacitante, ha ido reduciéndose hasta quedar reservada para los casos más complejos. Los avances en el tratamiento farmacológico y la rehabilitación cardiaca han mejorado también la prevención secundaria de los pacientes isquémicos, evitando recaídas y mejorando la capacidad funcional, facilitando de esta forma la reincorporación laboral ${ }^{33,34}$.

En el Registro Español de Hemodinámica y Cardiología Intervencionista de la Sección de Hemodinámica y Cardiología Intervencionista de la Sociedad Española de Cardiología se observa la evolución del número de intervenciones coronarias percutáneas realizadas en España durante el período 1999 al 2012 ${ }^{32}$. En el informe se observa un crecimiento progresivo de la angioplastia en el contexto del infarto agudo, en particular la ICP primaria como procedimiento de revascularización coronaria, lo que implica una clara y progresiva mejora asistencial del síndrome coronario agudo en nuestro país.

Con respecto a la Incapacidad Temporal (I. T.) la duración media de los procesos continúa siendo muy alta (en torno a 202 días de media). No obstante es menor que la publicada en estudios previos (243 días de media de duración de IT en el estudio de 
Gutiérrez Morlote et al. ${ }^{9}$ y 222 días de media en el estudio de Delclós et al. para el período de $2002-2006{ }^{40}$ ) y claramente superior a la duración media de los países de nuestro entorno en los que mayoritariamente se reincorporan antes de los 6 meses ${ }^{25,37}$. Probablemente la descoordinación entre niveles asistenciales puede ser una causa importante responsable de esta prolongación de la I. T. En la mayoría de los casos es el médico de atención primaria quien da los partes de baja y en su momento el alta. Por lo general, los médicos de atención primaria no suelen dar el alta laboral hasta que el paciente no ha pasado la primera consulta de revisión por el cardiólogo de referencia. Esto conlleva un alargamiento, innecesario en la mayoría de los pacientes que evolucionan de forma asintomática, de los tiempos de baja.

No obstante, en nuestro estudio se observa una clara mejoría progresiva y continúa en la última década. (Desde 259 días en el año 2000 a una media de 170 días en 2011). La reducción de los costes sanitarios por este concepto se ha convertido en un objetivo prioritario de empresas y administración pública. Una muestra de ello es la elaboración de guías que reflejan estándares de duración de la I. T según el diagnóstico clínico. En España el Instituto Nacional de Seguridad Social (INSS) publicó recientemente una lista de tiempos estándar de duración de la IT para cada uno de los procesos diagnósticos ${ }^{38}$ No cabe duda de que además de las razonas anteriormente expuestas (mejoría de las intervenciones terapéuticas), estas guías están siendo aportaciones útiles en la mejora de la gestión de los procesos tanto para los clínicos como para los gestores de la de la IT $^{39}$.

\section{CONCLUSIONES}

El estudio que presentamos, tanto por su tamaño muestra (5067 pacientes) como por el ámbito geográfico (país España) como por el intervalo de tiempo en el que se desarrolla (recoge las importantes mejoras en el tratamiento y prevención de la cardiopatía isquémica desarrolladas en los últimos años) consideramos que supone una de las fuentes más importante de datos para el estudio clínico y la gestión de la I. P e I. T en nuestro país.

En la última década se ha observado en nuestro país una reducción significativa año tras año de la incapacidad laboral por cardiopatía isquémica, desde el 33\% en el año 2000 a $<10 \%$ en el 2010 , de modo que en nuestros días un porcentaje importante de los pacientes con infarto se reincorporan a su trabajo, especialmente los pacientes más jóvenes, y los que desempeñan una ocupación administrativa o profesional.

En cambio, la cardiopatía isquémica sigue generando un largo período de incapacidad temporal, que no se corresponde con variables clínicas ni los avances terapéuticos o mejoría del pronóstico de la cardiopatía isquémica experimentado en las dos últimas décadas.

La extensión del daño miocárdico (medido por la FEVI) así como el procedimiento de revascularización (ACTP vs Cirugía), el régimen laboral (autónomo vs cuenta ajena), tipo de ocupación, retribución salarial y la edad (especialmente ser mayor de 50 años) son los mejores predictores de la reincorporación laboral de pacientes que causan baja por cardiopatía isquémica.

\section{REFERENCIAS BIBLIOGRÁFICAS}

1. Instituto Nacional de Estadística. Defunciones según la Causa de Muerte. Año 2007. Madrid: Instituto Nacional de Estadística; 2009

2. Estrategia en Cardiopatía Isquémica del Sistema Nacional de Salud. Actualización aprobada por el Consejo Interterritorial del Sistema Nacional de Salud el 22 de octubre de 2009. Ministerio de Sanidad, Política Social e Igualdad. Secretaría General Técnica Madrid. 2011

3. Bhattacharyya M. R., Perkins-Porras L., Whitehead D. L., Steptoe A., Psychological and clinical predictors of return to work after acute coronary syndrome European Heart Journal (2007) 28, 160-165 
4. O'Neil A., Sanderson K., Oldenburg B. Depression as a predictor of work resumption following myocardial infarction (MI): a review of recent research evidence Health and Quality of Life Outcomes 2010, 8:95.

5. Hämäläinen H, Mäki J, Virta L, Keskimäki I, Mähönen M, Moltchanov V, et al. Return to work after first myocardial infarction in 1991-1996 in Finland. Eur J Public Health. 2004;14:350-3.

6. Expósito Tirado J. A., Olmo Vega J. A, Rodríguez García J., Ferrand Ferri P., Echevarría Ruiz de Vargas C., Rodríguez-Piñero Durán M. Reincorporación laboral de los pacientes que han sufrido un infarto agudo de miocardio. MAPFRE MEDICINA Vol 17 N. 2 Enero/Marzo 2006.

7. Jiménez Sánchez M., Mata Escriche C., Bascuas Hernández J., Abad Domingo E., García Felipe A I., Rubio Calvo E., Reincorporación laboral del trabajador coronario: criterios objetivos para un cambio de puesto de trabajo. TRAUMA. Fundación Mapfre Vol 19 n. 3 Julio/Septiembre 2008.

8. García Ruiz F. J, Alonso Calderón M. I, Gómez González A. M, Montiel Trujillo A., Mora Robles J., Pérez Sánchez F., Reincorporación laboral en Málaga de trabajadores con cardiopatía, según el tipo de trabajo que realizan. Prevexpo 2010 - X Congreso Andaluz de Seguridad y Salud Laboral. Consejería de Empleo de la Junta de Andalucia.2010.

9. Gutiérrez Morlote J, Vacas Arlandis M, Lobato García A, Llorca Díaz J, Prieto Solís JA, Domenech Delgado J y San José Garagarza JM. Impacto del infarto de miocardio en la situación laboral de los pacientes Rev Esp Cardiol. 1999;52:556-62. Vol.52 Núm.

10. Ferreira-González I., Permanyer-Miralda G., Marrrugat J., Heras M., Cuñat J., Civeira E., Arós F., Rodríguez J. J, Sánchez P. Bueno H., Estudio MASCARA (Manejo del Síndrome Coronario Agudo. Registro Actualizado). Resultados globales Rev Esp Cardiol. 2008;61(8):803-16.

11. Dégano I. R., Elosua R., Marrugat J., Epidemiología del síndrome coronario agudo en España: estimación del número de casos y la tendencia de 2005 a 2049 Rev Esp Cardiol. 2013;66(6):472-481.

12. Sobrino JA., Merino JL., Maté I., García García J., L., Rico J., Plaza I., Oliver JM., Calvo L., Sobrino N. Return to work after coronary revascularization Rev Esp Cardiol. 1995 Mar;48(3):183-6.

13. Worcester M, Elliott P, Turner A, Pereira J, Murphy B, Le Grande M, Middleton K, Navaratnam H, Nguyen J, Newman R, Tatoulis J. Resumption of work after acute coronary syndrome or coronary artery bypass graft surgery. Heart Lung \& Circulation 2014; 23:444-453.

14. Abbas A. E., Brodie B., Stone G., Cox D., Berman A., Brewington S., Dixon S., O,Neill W. W, Grines C. L., Frequency of returning to work one and six months following percutaneous coronary intervention for acute myocardial infarctionThe American Journal of Cardiology Volume 94, Issue 11, 1 December 2004, Pages 1403-1405.

15. Boudrez H, De Backer G. Recent findings on return to work after an acute myocardial infarction or coronary artery bypass grafting. Acta Cardiol. 2000 Dec;55(6):341-9.

16. Boudrez H, De Backer G, Comhaire B. Return to work after a myocardial infarction: results of a longitudinal population based study. Eur Heart J 1994;15:32-6.

17. Isaaz K., Coudrot M., Sabry, M., Cerisier A., Lamaud M., Robin C., Richard L., da Costa A., Khamis H., Abd-Alaziz A, Gerenton C., Return to work after acute ST-segment elevation myocardial infarction in the modern era of reperfusion by direct percutaneous coronary intervention. Archives of Cardiovascular Disease (2010) 103, 310-316.

18. Mittag O., Kolendab K. O, Nordmanna K. J, Bernien J., Maurischat C.: Return to work after myocardial infarction/coronary artery bypass grafting: patients $>$ and physicians $>$ initial viewpoints and outcome 12 monthslater Social Science \& Medicine 52 (2001) 1441-1450.

19. Waszkowska M., and Szymczak W., Return to work after myocardial infarction: A restrospective study. International Journal of Occupational Medicine and Environmental Health 2009;22(4):373-381.

20. Farkas JP, Cerne K, Lainscak M, Keber I. Return to work after myocardial infarction-listen to your doctor! Inter J Cardiol 2008;130: e14-6.

21. Petrie KJ, Weinman J, Sharpe N, Buckley J. Role of patients > view of their illness in predicting return to work and functioning after myocardial infarction: longitudinal study. BMJ 1996;312:1191-1194.

22. Soejima Y, Steptoe A, Nozoe S, Tei C. Psychosocial and clinical factors predicting resumption of work following acute myocardial infarction in Japanese men. Int J Cardiol 1999;72:39-47.

23. Fonager K., Lundbye-Christensen S., Andreasen J. J, Futtrup M., Luther Christensen A., Ahmad K., Agge Nørgaard M.: Work Status and Return to the Workforce after Coronary Artery Bypass Grafting and/or Heart Valve Surgery: A One-Year-Follow Up StudyHindawi Publishing Corporation Rehabilitation Research and Practice Volume 2014, Article ID 631842.

24. Sellier Ph., Varaillac P et al. Factors influencing return to work at one year after coronary bypass graft surgery: results of the PERISCOP study. Eur J Cardiovasc Prevention Rehab 2003; 10: 469-475. 
25. Hällberg V., Palomäki A., Kataja M. et al., "Working after CABG study group. Return to work after coronary artery bypass surgery. A 10-year follow-up study," Scand Cardiovasc, vol. 43, no. 5, pp. 277-284, 2009.

26. Speziale G, Bilotta F, Ruvolo G, et al. Return to work and quality of life measurement in coronary artery bypass grafting. Eur J Cardiothorac Surg 1996;10:852-858.

27. Real Decreto 818/2009, de 8 de mayo, por el que se aprueba el Reglamento General de Conductores. MINISTERIO DEL INTERIOR BOE núm. 138 de 08 de Junio de 2009.

28. Duran X., Martínez J. M., Benavides F. G., Tendencia temporal de la incapacidad laboral en España (19922010) Rev Esp Salud Pública 2012; 86: 533-542.

29. Benavides F. G., Durán X., Martínez J. M., Jódar P., Boix P., Amable M.,: Incidencia de incapacidad permanente en una cohorte de trabajadores afiliados a la Seguridad Social, 2004-2007 Gac Sanit.2010;24 (5):385-390.

30. Alba Ramírez A. La Incapacidad temporal para el trabajo Análisis económico de su incidencia y duración. Universidad Carlos III de Madrid. Ministerio de Trabajo e Inmigración. Secretaría de Estado de la Seguridad Social Madrid 2009.

31. García del Blanco B., Rumoroso Cuevas J. R., Hernández Hernández F., Trillo Nouche R., Registro Español de Hemodinámica y Cardiología Intervencionista. XXII Informe Oficial de la Sección de Hemodinámica y Cardiología Intervencionista de la Sociedad Española de Cardiología (1990-2012) Rev Esp Cardiol. 2013;66(11):894-904.

32. García-García C., Sanz G., Vallee V., Molina L., Salag J., Subirana I., Martí H., Marrugat J., Bruguera J., Masiàg R., Elosua R., Evolución de la mortalidad intrahospitalaria y el pronóstico a seis meses de los pacientes con un primer infarto agudo de miocardio. Cambios en la última década Rev Esp Cardiol. 2010;63(10):1136-44.

33. Maroto Montero JM, Artiago Ramírez R, Morales Durán MD, De Pablo Zarzosa C, Abraira V. Rehabilitación cardiaca en pacientes con infarto de miocardio. Resultado tras 10 años de seguimiento. Revista Española de Cardiología 2005; 58:1181-1187.

34. Álvarez-Bandrés N., Maillos-Torán M., Domínguez Aragó A., Lapresta C., Numancia-Andreu R., Reinserción laboral tras infarto agudo de miocardio (IAM): tratamiento rehabilitador frente a tratamiento convencional tras IAM Rehabilitación (Madrid). 2008;42(5):224-30.

35. Sellier $\mathrm{Ph}$., Varaillac $\mathrm{P}$ et al. Factors influencing return to work at one year after coronary bypass graft surgery: results of the PERISCOP study. Eur J Cardiovasc Prevention Rehab 2003; 10: 469-475.

36. Picard $\mathrm{MH}$, Dennos $\mathrm{CH}$ et al. Cost-benefit analysis of early return to work after uncomplicated acute myocardial infarction. Am J Cardiol 1989; 63: 1308-1314.

37. Ministerio de Trabajo e Inmigración. Secretaría de Estado de la Seguridad Social. Tiempos estándar de incapacidad temporal [citado 12 nov 2009]. Disponible en: http://www.seg-social.es/prdi00/groups/ public/documents/binario/ 122970.pdf.

38. Sanz Carabaña P., Flores Ortueta L., Soto Fernández S., Villagrasa Ferrer J. R., Berriocochoa Martínez de Pison C.: Utilidad de la guía de estándares de duración de procesos de incapacidad laboral transitoria publicada por el Insalud para el médico de cabecera. Atención Primaria. 1996; 18: 248-52.

39. Delclós J., García S., López J. C., Sampere M., Serra C., Plana M., Vila D., Benavides F. G., Duración de la incapacidad temporal por contingencia común por grupos diagnósticos. Arch Prev Riesgos Labor 2010; 13 (4): $180-187$.

|| 